Como citar este artigo:

MAIER, SUELLEN R. O.; BAZZANO, ANNA BEATRIZ K. R. M.; OLIVEIRA,

UANMAR S.; CORRÊA, CARLA

REGINA A.; SOARES JÚNIOR,

JOAQUIM R:; SUDRÉ, MAYARA

R. S.; MOSER, GELSON A. S.;

ACUIAR, DENISE C. M.; SUDRÉ,

CRACIANO A.; Fatores de riscos

relacionados ao infarto agudo

do miocárdio: revisão integrativa da literatura. Revista Saúde (Sta. Maria). 2020; 46 (1).

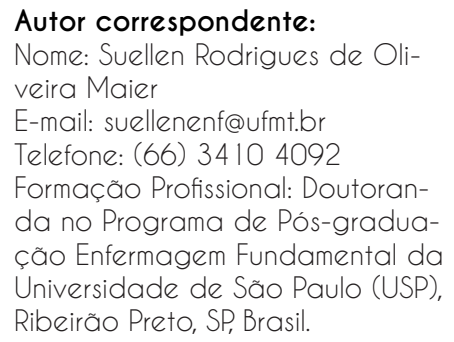

Filiação Institucional: Universidade Federal de Rondonópolis Endereço para correspondência: Rua: Avenida dos Estudantes $n^{\circ}$ 5055

Bairro: Cidade Universitária

Cidade: Rondonópolis

Estado: Mato Grosso

CEP: 78700-000

Data de Submissão:

23/03/2020

Data de aceite:

26/03/2020

Conflito de Interesse: Não há

conflito de interesse

\section{Fatores de riscos relacionados ao infarto agudo do miocárdio: revisão integrativa da literatura}

\author{
Risk factors related to acute myocardial infarction \\ myocardium: integrative literature review
}

Suellen Rodrigues de Oliveira Maier, Anna Beatriz Kétsia Ribeiro Moreira Bazzano, Wanmar de Souza Oliveira, Carla Regina de Almeida Corrêa, Joaquim Rosa Soares Júnior, Mayara Rocha Siqueira Sudré, Gelson Aguiar da Silva Moser, Denise Consuelo Moser Aguiar, Graciano Almeida Sudré

\section{RESUMO}

Analisar evidencias cientificas, relacionado aos fatores de riscos para o ocorrência de Infarto do Miocárdio na literatura. Método: Trata-se de uma revisão integrativa da literatura realizada em bases de dados: LILACS, MEDLINE/PubMed, SCOPUS, BDENF e Web of Science, tendo como critérios de elegibilidade artigos de pesquisa publicados entre os anos de 2013 e 2017, nos idiomas português, inglês e espanhol, disponíveis na íntegra e que respondessem a questão norteadora, sendo selecionados nove artigos. Resultados: ao témino das buscas, nove artigos foram selecionados. Foram evidenciados como fatores de risco: diabetes mellitus, tabagismo e hipertensão arterial. Em contrapartida, a periodontite, o lúpus eritematoso sistêmico, a Doença do Refluxo Gastroesofágico, a menor secreção de melatonina, a depressão, a enxaqueca e os aspectos emocionais são fatores de risco relevantes. Conclusão: Atráves deste estudo sugere-se que as vítimas de infarto do miocárdio apresentam fatores de riscos análogos, por exemplo, diabetes mellitus, tabagismo e hipertensão arterial. Todavia, outros estudos apresentaram outras comorbidades cardíacas ou não cardíacas, como favoráveis ao desenvolvimento do infarto.

PALAVRAS-CHAVE: Fatores de risco; Infarto do miocárdio; Unidades de Terapia Intensiva; Prevenção \& Controle; Doenças Cardiovasculares.

\section{ABSTRACT}

To analyze scientific evidence, related to the risk factors for the occurrence of Myocardial Infarction in the literature. Method: This is an integrative literature review carried out in databases: LILACS, MEDLINE / PubMed, SCOPUS, BDENF and Web of Science, with eligibility criteria research articles published between 2013 and 2017, in the languages Portuguese, English and Spanish, available in full and that answered the guiding question, with nine articles selected. Results: at the end of the searches, nine articles were selected. Risk factors were: diabetes mellitus, smoking and high blood pressure. In contrast, periodontitis, systemic lupus erythematosus, Gastroesophageal Reflux Disease, lower melatonin secretion, depression, migraine and emotional aspects are relevant risk factors. Conclusion: This study suggests that victims of myocardial infarction have similar risk factors, for example, diabetes mellitus, smoking and high blood pressure. However, other studies have shown other cardiac or noncardiac comorbidities, as favorable to the development of infarction.

KEYWORDS: Risk factors; Myocardial infarction; Intensive Care Units; Prevention \& Control; Cardiovascular Diseases. 


\section{INTRODUÇÃO}

As doenças cardiovasculares (DCV) são a principal causa de morte no mundo, representando $31 \%$ de todas as mortes em nível global. A maioria das DCVs podem ser prevenidas por meio de abordagem aos fatores comportamentais de risco, como por exemplo, o uso do tabaco, hábitos alimentares inadequados, obesidade, sedentarismo, e uso de álcool, utilizando estratégias para a população em geral' .

A doença arterial coronariana (DAC) constitui uma classe de DCV com altos índices de internações. Tal agravo permanece como uma das maiores causas de mortalidade nos países em desenvolvimento, alcançando incidência e prevalência epidêmicas em vários locais do mundo, sendo que o Infarto Agudo do Miocárdio (IAM) representa 12,1\% das mortes por DCV 2,3 .

Dentre as DAC destacam-se as síndromes coronarianas agudas (SCA), sendo estas caracterizadas por várias manifestações clínicas e classificadas em três formas: Angina Instável (AI), Infarto Agudo do Miocárdio (IAM) sem supradesnível do segmento ST e IAM com supradesnível do segmento ST. Considera-se Infarto Agudo do Miocárdio a morte de um segmento do músculo cardíaco por falta de irrigação sanguínea, esta por sua vez é causada por ruptura de ateroma ou trombo, resultando em obstrução parcial ou completa da artéria. Este processo desencadeia um desequilíbrio entre oferta e demanda de oxigênio ao miocárdio, levando ao Infarto do Miocárdio ${ }^{4,5}$.

Os fatores de risco associados ao desenvolvimento do IAM, estão em destaque à idade acima de 55 anos, sexo masculino, história familiar de DCV, dislipidemia (DLP), tabagismo, hipertensão arterial sistêmica (HAS), sedentarismo, obesidade, diabetes mellitus (DM), hábitos alimentares inadequados e estresse psicossocial.

Os fatores de risco têm sido preponderantes para o aumento da mortalidade por IAM no cenário brasileiro, sendo observado o aumento de 48\% entre 1996 e $2011^{3,6}$. O conhecimento relacionados aos fatores de riscos na população brasileira se torna decisivo para a adoção de medidas preventivas eficazes no contexto do cuidado em saúde em todos os pontos da rede de atenção.

Diante deste contexto, este estudo tem como objetivo verificar os principais fatores de riscos relacionados à ocorrência de Infarto Agudo do Miocárdio referenciados na literatura.

\section{MÉTODO}

Trata-se de uma revisão integrativa da literatura, com o intuito de reunir os resultados de estudos desenvolvidos mediante diferentes métodos, permitindo aos autores sintetizar resultados sem ferir a filiação epistemológica dos estudos empíricos incluídos. As fases relacionadas ao método de estudo foram distribuídas em caráter organizacional: Fase | - identificação do tema e/ou questão de pesquisa; Fase II - estabelecimento de critérios para inclusão e exclusão dos estudos e busca sistematizada na literatura; Fase III - definição das informações a serem extraídas dos estudos selecionados conforme a questão norteadora da revisão; Fase IV - avaliação dos estudos incluídos na revisão; Fase V - 
interpretação dos resultados encontrados; Fase VI - síntese do conhecimento ${ }^{7}$.

A fase de seleção dos estudos ocorreu por meio de busca eletrônica a artigos originais que respondessem à pergunta de investigação, sendo utilizada a estratégia PICO (Paciente, Intervenção, Controle ou Comparação e Outcomes - Desfecho) para a construção: Quais são os fatores de risco que ocasionam o desenvolvimento do infarto agudo do miocárdio apontados na literatura? Compuseram a amostra artigos publicados na íntegra advindos de estudos de coorte e caso-controle, em português, inglês e espanhol, publicados de 2013 a 2017, que respondessem à pergunta de investigação. Foram excluídos artigos com inconsistência metodológica e estatística.

As bases de dados eletrônicas acessadas foram: Literatura Latino-Americana e do Caribe em Ciências da Saúde (LILACS), Medical Literature Analysis and Retrieval System Online (MEDLINE), SCOPUS, Base de dados em Enfermagem (BDENF) e Web of Science. Os descritores utilizados foram submetidos na Biblioteca Virtual em Saúde, Descritores em Ciências da Saúde e no Medical SubjectHeading (MeSH): "infarto do miocárdio", "infarto do miocárdio" e "fatores de risco", "infarto do miocardio" e "unidades de terapia intensiva", "myocardialinfarction" e "riskfactors" e "myocardialinfarction" e"intensivecareunit". Todos os cruzamentos foram realizados utilizando o operador booleano AND.

Ao final da seleção os estudos de coorte e caso-controle foram avaliados quanto à qualidade metodológica, utilizando a escala Newcastle-Ottawa. A pontuação dos estudos foi calculada em três domínios: seleção dos grupos (0 4 pontos), comparabilidade ( 0 - 2 pontos $)$ e resultados ( 0 - 3 pontos). A pontuação máxima pode ser de 9 pontos, 0 que representa alta qualidade metodológica. Os artigos que apresentarem pontuação inferior a 4, indicam evidência limitada ou baixa qualidade, sendo assim excluídos ${ }^{8}$.

Quadro I. Cruzamento dos descritores por base de dados.

\begin{tabular}{|c|ll|}
\hline Base de dados & \multicolumn{1}{c|}{ Cruzamento dos descritores } \\
\hline BDENF & $x$ & "risk factors" AND "myocardial infarction" \\
& $x$ & "myocardial infarction" AND "intensive care unit" \\
& $x$ & "myocardial infarction" AND "intensive care unit" AND "risk factors" \\
& $x$ & "infarto do miocárdio" AND "fatores de risco" \\
& $x$ & "infarto do miocardio" AND "unidades de terapia intensiva" \\
& $x$ & "infarto do miocárdio" AND "fatores de risco" AND "unidades de terapia intensiva" \\
\hline LILACS & $x$ & "infarto do miocárdio" AND "fatores de risco" \\
& $x$ & "infarto do miocardio" AND "unidades de terapia intensiva" \\
\hline MEDLINE & $x$ & "infarto do miocárdio" AND "fatores de risco" AND "unidades de terapia intensiva" \\
& $x$ & "risk factors" AND "myocardial infarction" \\
& $x$ & "myocardial infarction" AND "intensive care unit" \\
\hline SCOPUS & $x$ & "risk factors" AND "myocardial infarction" \\
& $x$ & "myocardial infarction" AND "intensive care unit" \\
& $x$ & "myocardial infarction" AND "intensive care unit" AND "risk factors" \\
\hline Web of Science & $x$ & "risk factors" AND "myocardial infarction" \\
& $x$ & "myocardial infarction" AND "intensive care unit" \\
& $x$ & "myocardial infarction" AND "intensive care unit" AND "risk factors" \\
\hline
\end{tabular}

Fonte: dados da pesquisa. 2019. 
Para a descrição do estudo, utilizou-se o fluxograma Preferred Reporting Items for Systematic Review and MetaAnalyses (PRISMA) ${ }^{7,9}$ que norteou a busca e seleção dos estudos, conforme a figura 1.

Figura 1 - Fluxograma de identificação, seleção e inclusão dos estudos da revisão integrativa da literatura. Rondonópolis (MT), Brasil, 2018.

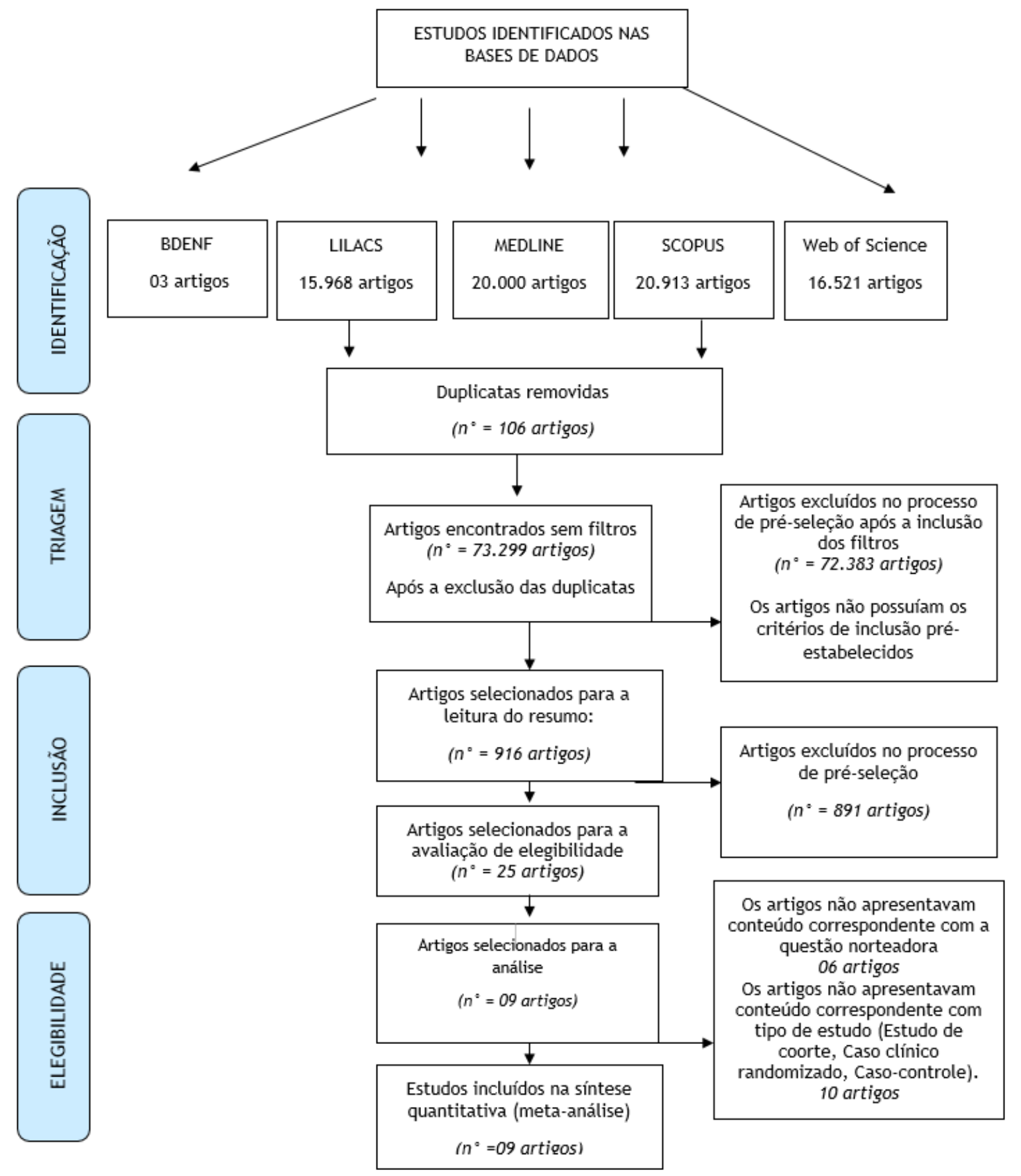

Fonte: dados da pesquisa.

Os cruzamentos e a análise de dados ocorreram nos meses de novembro e dezembro de 2018 e janeiro de 2019 pelos pesquisadores de forma independente, e para a coleta de dados os descritores foram inseridos nas bases eletrônicas, previamente, respeitando os critérios de inclusão e exclusão descritos. Após esse processo os artigos foram 
selecionados por intermédio da leitura dos títulos que apresentavam correlação com o tema principal.

Posteriormente à análise dos títulos, os artigos foram antepostos por meio de uma leitura criteriosa de seus resumos e aqueles que se aproximavam do assunto central proposto pela pergunta norteadora foram escolhidos para leitura na íntegra. Para a consolidação realizou-se leitura e avaliação, restando nove artigos. Nesta perspectiva, os achados permitiram elencar os fatores de risco evidentes nos estudos internacionais relacionados ao desenvolvimento do infarto agudo do miocárdio.

\section{RESULTADOS}

Após o término das buscas nas bases de dados eletrônicas citadas anteriormente com a utilização dos critérios de inclusão descritos previamente, e após a análise da qualidade metodológica, os achados foram sistematizados a partir de dois quadros.

Quadro II: Caracterização dos estudos selecionado nas base de dados, conforme autores, título, periódico, local de estudo e Escala de Newcastle-Ottawa. Rondonópolis, MT, Brasil, 2019.

\begin{tabular}{|c|c|c|c|c|c|c|}
\hline & $\begin{array}{l}\text { Base de } \\
\text { dados }\end{array}$ & Autores & Título & Periódico & Local & $\begin{array}{l}\text { Escala de } \\
\text { Newcastle- } \\
\text { Ottawa }\end{array}$ \\
\hline $\mathrm{A} 1$ & LILACS & $\begin{array}{l}\text { Cortés et al, } \\
2013^{10}\end{array}$ & $\begin{array}{l}\text { Factores de riesgo coronarios asociados } \\
\text { al infarto agudo Del miocardio en el adulto } \\
\text { mayor }\end{array}$ & $\begin{array}{l}\text { Centro Provin- } \\
\text { cial de Ciências } \\
\text { Médicas - Me- } \\
\text { disan. }\end{array}$ & Cuba & Score 07 \\
\hline A2 & MEDLINE & $\begin{array}{l}\text { Górski et al, } \\
2016^{11}\end{array}$ & $\begin{array}{c}\text { The Association Between Dental Status and } \\
\text { Risk of Acute Myocardial Infarction Among } \\
\text { Poles: Case-control Study }\end{array}$ & $\begin{array}{l}\text { Wroclaw Medi- } \\
\text { cal University } \\
\text { - Adv Clin Exp } \\
\quad \text { Med. }\end{array}$ & Polônia & Score 08 \\
\hline A3 & MEDLINE & $\begin{array}{l}\text { Avina-Zu- } \\
\text { bieta et al, } \\
2017^{12}\end{array}$ & $\begin{array}{l}\text { Risk of Myocardial Infarction and Stroke in } \\
\text { Newly Diagnosed Systemic Lupus Erythema- } \\
\text { tosus: A General Population-Based Study }\end{array}$ & $\begin{array}{l}\text { Arthritis Care \& } \\
\text { Research. }\end{array}$ & Canada & Score 06 \\
\hline A4 & MEDLINE & $\begin{array}{l}\text { Lei et al, } \\
2017^{13}\end{array}$ & $\begin{array}{l}\text { Risk of acute myocardial infarction in patients } \\
\text { with gastroesophageal reflux disease: A } \\
\text { nationwide population-based study }\end{array}$ & Plosone. & Taiwan & Score 08 \\
\hline A5 & MEDLINE & $\begin{array}{l}\text { McMullan et } \\
\text { al, } 2016^{14}\end{array}$ & $\begin{array}{l}\text { A nested case-control study of the associa- } \\
\text { tion } \\
\text { between melatonin secretion and incident } \\
\text { myocardial infarction }\end{array}$ & The BMJ & $\begin{array}{l}\text { Estados } \\
\text { Unidos }\end{array}$ & Score 08 \\
\hline A6 & MEDLINE & $\begin{array}{l}\text { Jernberg et } \\
\text { al, } 2015^{15}\end{array}$ & $\begin{array}{c}\text { Cardiovascular risk in post-myocardial } \\
\text { infarction patients: nationwide real world data } \\
\text { demonstrate the importance of a long-term } \\
\text { perspective }\end{array}$ & $\begin{array}{c}\text { European Heart } \\
\text { Journal }\end{array}$ & Suécia & Score 07 \\
\hline A7 & MEDLINE & $\begin{array}{l}\text { Sundbø\|l et } \\
\text { al, } 2017^{16}\end{array}$ & $\begin{array}{l}\text { Impact of pre-admission depression on } \\
\text { mortality } \\
\text { Followingmyocardialinfarction }\end{array}$ & $\begin{array}{l}\text { The British Jour- } \\
\text { nal of Psychiatry }\end{array}$ & Dinamarca & Score 09 \\
\hline
\end{tabular}




\begin{tabular}{|c|c|c|c|c|c|c|}
\hline A8 & MEDLINE & $\begin{array}{c}\text { Aldeborg et } \\
\text { al, } 2017^{17}\end{array}$ & $\begin{array}{c}\text { Migraine and risk of cardiovascular diseases: } \\
\text { Danish population based matched cohort } \\
\text { study }\end{array}$ & The BMJ & Dinamarca & Score 09 \\
\hline A9 & SCOPUS & $\begin{array}{c}\text { Ghiasmand } \\
\text { et al, } 2017^{18}\end{array}$ & $\begin{array}{c}\text { Acute triggers of myocardial infarction: A } \\
\text { case-crossover study }\end{array}$ & $\begin{array}{c}\text { The Egyptian } \\
\text { Heart Journal }\end{array}$ & Irã & Score 08 \\
\hline
\end{tabular}

Fonte: Dados da pesquisa.

Quadro III: Caracterização dos estudos de acordo com autores, objetivo, tipo de estudo, população do estudo e fatores de risco. Rondonópolis, MT, Brasil, 2019

\begin{tabular}{|c|c|c|c|c|c|}
\hline & Autores & Objetivo & $\begin{array}{l}\text { Tipo de } \\
\text { Estudo }\end{array}$ & $\begin{array}{c}\text { População do } \\
\text { Estudo }\end{array}$ & Fatores de Risco \\
\hline A1 & $\begin{array}{l}\text { Cortés et al, } \\
2013^{10}\end{array}$ & $\begin{array}{l}\text { Identificar os fatores de risco co- } \\
\text { ronariano associados ao Infarto } \\
\text { Agudo do Miocárdio }\end{array}$ & Caso-controle & $\begin{array}{l}\text { Idosos com infarto } \\
\text { agudo do miocár- } \\
\text { dio, no período de } \\
\text { janeiro de } 2006 \text { a } \\
\text { dezembro de } 2011 . \\
\text { Foram selecio- } \\
\text { nados } 33 \text { partici- } \\
\text { pantes, sendo } 22 \\
\text { controle e } 11 \text { caso. }\end{array}$ & $\begin{array}{l}\text { Hipertensão arterial }(69,7 \% \text { casos } \\
\text { para } 37,9 \text { controles; OR } 3,8 ; \text { IC } \\
\text { 1,4-10,2); } \\
\text { Sedentarismo (84,9\% casos para } \\
62,1 \% \text { controles; OR 3,4; IC 1,1- } \\
11,6) ; \\
\text { Tabagismo (60,6\% dos casos, e } \\
34,8 \% \text { dos controles; OR 2,9; IC } \\
\text { 1,1-7,5); } \\
\text { Diabetes Mellitus (39,4\% casos } \\
\text { para 27,3\%controles; OR 1,7; IC } \\
\text { 0,7-4,6); } \\
\text { Sobrepeso (36,4\% casos para } \\
37,9 \% \text { controles; OR 0,9). }\end{array}$ \\
\hline $\mathrm{A} 2$ & $\begin{array}{l}\text { Górski et al, } \\
2016^{11}\end{array}$ & $\begin{array}{l}\text { Investigar a associação da gravi- } \\
\text { dade e extensão da periodontite } \\
\text { com infarto agudo do miocárdio. }\end{array}$ & Caso controle & $\begin{array}{l}134 \text { casos hospita- } \\
\text { lizados com infarto } \\
\text { agudo do miocárdio } \\
\text { com idade inferior } \\
\text { a } 70 \text { anos e } 155 \\
\text { controles. }\end{array}$ & $\begin{array}{l}\text { Periodontite }(\mathrm{OR}=2,0 ; \mathrm{IC} 95 \%= \\
1,2-3,5 ; p=0,0109) ; \\
\text { Sexo Masculino }(\mathrm{OR}=5,6) ; \\
\text { Tabagismo (OR=11,1); } \\
\text { Hipertensão Arterial }(\mathrm{OR}=6,6) ; \\
\text { Diabetes }(\mathrm{OR}=3,8) ; \\
\text { Obesidade }(\mathrm{OR}=3,1) ; \\
\text { Sobrepeso }(\mathrm{OR}=2,0)\end{array}$ \\
\hline A3 & $\begin{array}{l}\text { Avina-Zubieta } \\
\text { et al, } 2017^{12}\end{array}$ & $\begin{array}{l}\text { Estimar o risco futuro e as ten- } \\
\text { dências temporais do infarto do } \\
\text { miocárdio recentemente diagnos- } \\
\text { ticado, acidente vascular cerebral } \\
\text { isquêmico ou ambos (doença } \\
\text { cardiovascular) em indivíduos } \\
\text { com lúpus eritematoso sistêmico. }\end{array}$ & $\begin{array}{l}\text { Estudo de } \\
\text { coorte }\end{array}$ & $\begin{array}{l}4.863 \text { indivíduos } \\
\text { com lúpus eritema- } \\
\text { toso sistêmico. }\end{array}$ & $\begin{array}{l}\text { Lúpus eritematoso sistêmico } \\
(\mathrm{RR}=6,4, \mathrm{IC} 95 \%) \text {. }\end{array}$ \\
\hline A4 & $\begin{array}{l}\text { Lei et al, } 2017 \\
13\end{array}$ & $\begin{array}{l}\text { Comparar a incidência de infarto } \\
\text { agudo do miocárdio em pacien- } \\
\text { tes com Doença do Refluxo Gas- } \\
\text { troesofágico (DRGE) com idade, } \\
\text { sexo e comorbidade população } \\
\text { compatível livre de DRGE }\end{array}$ & $\begin{array}{l}\text { Estudo de } \\
\text { coorte }\end{array}$ & $\begin{array}{l}54.422 \text { pacientes } \\
\text { recém-diagnostica- } \\
\text { dos com DRGE }\end{array}$ & $\begin{array}{l}\text { DRGE (OR=1,48; IC95\%: 1,31- } \\
1,66 \text {, p-valor <0,001). }\end{array}$ \\
\hline A5 & $\begin{array}{l}\text { McMullan et } \\
\text { al, } 2016^{14}\end{array}$ & $\begin{array}{l}\text { Estudar a associação entre a } \\
\text { secreção de melatonina e o } \\
\text { risco de desenvolver infarto do } \\
\text { miocárdio. }\end{array}$ & Caso-controle & $\begin{array}{l}209 \text { casos e } 209 \\
\text { controles. }\end{array}$ & $\begin{array}{l}\text { Menor secreção de Melatonina } \\
(\text { OR 1,51). }\end{array}$ \\
\hline
\end{tabular}




\begin{tabular}{|c|c|c|c|c|c|}
\hline A6 & $\begin{array}{l}\text { Jernberg et al, } \\
2015^{15}\end{array}$ & $\begin{array}{l}\text { Examinar o risco de eventos } \\
\text { cardiovasculares subsequentes } \\
\text { em pacientes que receberam alta } \\
\text { após o IM na Suécia. }\end{array}$ & \begin{tabular}{|l} 
Estudo de \\
coorte
\end{tabular} & $\begin{array}{l}108.315 \text { pacientes } \\
\text { admitidos no hos- } \\
\text { pital com infarto do } \\
\text { miocárdio primário } \\
\text { entre } 2006 \text { e } 2011 \\
\text { na Suécia }\end{array}$ & $\begin{array}{l}\text { Idade 60-69 anos: OR (IC95\%): } \\
\text { 1,37; } \\
\text { Idade 70-79 anos: OR 2,13; } \\
\text { Idade > } 80 \text { anos: OR 3,96; } \\
\text { Diabetes OR: 1,37; } \\
\text { Insuficiência Cardíaca OR: 1,57. }\end{array}$ \\
\hline A7 & $\begin{array}{l}\text { Sundboll et al, } \\
2017^{16}\end{array}$ & $\begin{array}{l}\text { Examinar a associação entre } \\
\text { depressão e mortalidade por } \\
\text { todas as causas após infarto do } \\
\text { miocárdio. }\end{array}$ & $\begin{array}{l}\text { Estudo de } \\
\text { coorte }\end{array}$ & $\begin{array}{l}170.771 \text { Pacientes } \\
\text { com infarto do mio- } \\
\text { cárdio pela primeira } \\
\text { vez entre } 1995 \text { a } \\
2014 \\
\end{array}$ & $\begin{array}{l}\text { Depressão RR 1,11, aumentando } \\
\text { para } 1,22 \text { quando se incluiu o uso } \\
\text { de antidepressivos. }\end{array}$ \\
\hline A8 & $\begin{array}{l}\text { Adelborg et al. } \\
2017^{17}\end{array}$ & $\begin{array}{l}\text { Examinar os riscos de infarto } \\
\text { agudo do miocárdio, acidente } \\
\text { vascular cerebral (isquêmico e } \\
\text { hemorrágico), a doença arterial } \\
\text { periférica, tromboembolismo } \\
\text { venoso, fibrilação atrial ou flutter } \\
\text { atrial, e insuficiência cardíaca em } \\
\text { pacientes com enxaqueca em } \\
\text { comparação com a população } \\
\text { geral. }\end{array}$ & \begin{tabular}{|l} 
Estudo de \\
coorte
\end{tabular} & $\begin{array}{l}51.032 \text { pacientes } \\
\text { com enxaqueca e } \\
510.320 \text { pessoas } \\
\text { da população geral } \\
\text { em todos os hospi- } \\
\text { tais dinamarqueses } \\
\text { e ambulatórios no } \\
\text { período de } 1995 \text { a } \\
2013 \text {. }\end{array}$ & $\begin{array}{l}\text { Enxaqueca (OR 1,49; IC 95\%: } \\
1,36-1,64) \text {. }\end{array}$ \\
\hline A9 & $\begin{array}{l}\text { Ghiasmand et } \\
\text { al, } 2017^{18}\end{array}$ & $\begin{array}{l}\text { Identificar as dimensões emo- } \\
\text { cionais, ambientais, físicas e } \\
\text { químicas de gatilhos agudos em } \\
\text { pacientes com IAM. }\end{array}$ & $\begin{array}{l}\text { Estudo de } \\
\text { coorte }\end{array}$ & $\begin{array}{l}269 \text { pacientes com } \\
\text { IAM, hospitalizados } \\
\text { em dois centros } \\
\text { de tratamento em } \\
\text { Rasht em } 2015\end{array}$ & 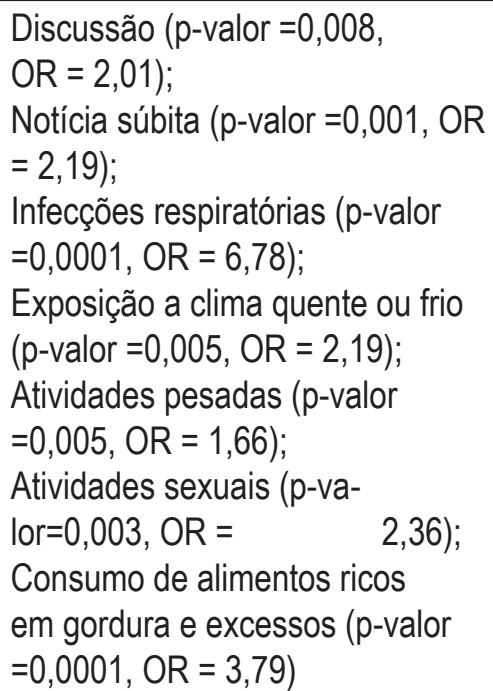 \\
\hline
\end{tabular}

Fonte: dados da pesquisa.

Legenda: RR: Risco Relativo; OR: OddsRatio; IC: Intervalo de Confiança.

Os dados nos quadros II e III explicitaram a caracterização dos estudos selecionados na fase de busca e foram confeccionados a fim de converter a sua essência, de modo a facilitar a compreensão dos legentes, evidenciando por intermédio das suas colunas a ligação dos resultados de forma dinâmica.

Quanto aos países de realização dos estudos, três foram produzidos no continente americano (um em Cuba, um no Canadá e um nos Estados Unidos), quatro em solo europeu (dois na Dinamarca, um na Suécia e um na Polônia) e dois estudos na Ásia (um no Irã e um em Taiwan). 


\section{DISCUSSÃO}

O infarto agudo do miocárdio, considerado um dos maiores eventos na evolução da cardiopatia isquêmica, é atualmente uma das principais causas de morte no mundo desenvolvido, contendo diversos fatores que o desencadeiam, sendo assim, é possível observar diferenças regionais e casuísticas nos estudos que compuseram este manuscrito.

Foram contemplados nos estudos fatores de riscos relacionados aos estilos de vida da população estudada, como, o sedentarismo e o tabagismo, bem como fatores patológicos pregressos, com a presença de outra doença como fator de risco para o desenvolvimento do infarto, como por exemplo, Lúpus Eritematoso Sistêmico ${ }^{12}$, Doença do Refluxo Gastroesofágico $^{13}$ e Doenças Crônicas Não Transmissíveis (Diabetes Mellitus ${ }^{10,11,15}$, Obesidade ${ }^{10,11,18}$, Hipertensão Arterial ${ }^{10,11}$, Insuficiência Cardíaca Congestiva ${ }^{15}$ ).

Os fatores relacionados ao envelhecimento evidenciaram que a população acima de sessenta anos ${ }^{15}$ está mais propensa a desenvolver eventos obstrutivos na musculatura cardíaca. O estudo realizado na Suécia ${ }^{15}$ revelou que o risco de eventos cardiovasculares está fortemente associado à idade e à presença de doenças crônicas (diabetes, hipertensão arterial, hábitos de vida em geral, IAM anterior, acidente vascular cerebral, angina instável ou insuficiência cardíaca) ${ }^{15}$.

No estudo realizado em Taiwan mostrou como fator de risco o DRGE, na qual a taxa de prevalência foi de cerca de 5,72\% na população do estudo, visto que a instilação de ácido para o esôfago pode reduzir, significativamente, 0 limiar de angina de esforço, provocando alterações do eletrocardiograma (ECG), consequentemente aumentando a predisposição para o IAM.Os autores ainda afirmam que a DRGE associada à hipertensão, diabetes, idade e tabagismo podem aumentar significativamente a ocorrência do IAM $^{13}$.

Em outro estudo realizado na Polônia a periodontite foi considerada um fator de risco singular para o IAM, contudo ao final da pesquisa conclui-se que a doença é somente considerada um fator desencadeante quando associada às doenças crônicas não transmissíveis, como por exemplo, a hipertensão, a obesidade, o tabagismo. No entanto, fatores como idade acima de sessenta anos e sexo masculino também possuem associação ${ }^{11}$.

Já no estudo dinamarquês, a enxaqueca esteve associada com o aumento dos riscos de várias doenças cardiovasculares em curto prazo. Como resultado, os autores expressam que 2451 pacientes tiveram um evento cardiovascular e 575 pacientes tinham mais de um evento cardiovascular. Igualmente, as incidências foram que 1000 pessoas após 19 anos de acompanhamento da enxaqueca, apresentaram 25\% de chances a mais para desencadear 0 $\mathrm{IAM}^{17}$.

Por outro lado, o estudo canadense, destacou que o Lúpus Eritematoso Sistêmico (LES) favorece o aumento do risco de IAM e outras doenças cardiovasculares. Os autores discursam que os altos índices de relação entre as doenças são decorrentes dos aspectos do LES, já que é uma doença autoimune sistêmica ${ }^{12}$. 
No decorrer da revisão alguns autores expressaram fatores de risco relevantes, entre eles a redução da secreção de melatonina, uma vez que os participantes com a secreção de melatonina inferior tinham significativamente maiores chances de desenvolver IAM, visto que a secreção de melatonina é responsável pela regulação do sono e bem-estar geral, ou seja, quando os níveis de melatonina estão reduzidos os aspectos biopsicossocioespirituais são afetados. 0 risco absoluto de infarto agudo do miocárdio na categoria mais elevada da secreção de melatonina foi estimado em 84 casos por 100.000 pessoas, achados epidemiológicos elencados no estudo norte americano ${ }^{14}$.

Em outro estudo dinamarquês, os pacientes com infarto do miocárdio com histórico de depressão anterior tinham uma mortalidade mais elevada não cardiovascular e mortalidade cardiovascular moderadamente maior do que os doentes sem depressão anterior ${ }^{16}$. Vários mecanismos fisiopatológicos subjacentes têm sido sugeridos para ligar depressão ao aumento da mortalidade em pacientes com infarto do miocárdio, e a causalidade é provável que seja multifatorial.

A evidência para uma via biológica sugere que a depressão pode estar associada à hiperatividade do eixo pituitário-adrenal hipotalâmico com níveis aumentados de cortisol, que pode levar à elevação da pressão sanguínea, aumento do volume de plasma, hiperinsulinemia, hiperglicemia, resistência à insulina e dislipidemia. É improvável que o aumento da mortalidade em decorrência do IAM possa ser atribuído ao tratamento antidepressivo ${ }^{16}$.

Por fim, em um estudo iraniano, aspectos de vida cotidiana ligados a relações interpessoais desajustadas, recebimento de notícias de forma súbita, infecção com foco respiratório, verão ou inverno local, atividades físicas extenuantes, atividades sexuais e hábitos alimentares inadequados (consumo de alimentos ricos em gorduras), cada fator de forma isolada podem favorecer ao desenvolvimento do $\operatorname{IAM}^{18}$. No entanto, os autores discorreram sobre a influência das doenças crônicas não transmissíveis existentes, referenciadas pelos participantes do estudo.

\section{CONSIDERAÇÕES FINAIS}

Ao longo da revisão integrativa da literatura ficou evidenciado que os autores internacionais expuseram os fatores de risco semelhantes aos nacionais, entretanto nota-se que há um déficit na produção nacional, uma vez que os fatores mais excêntricos foram encontrados em literaturas estrangeiras, como por exemplo, a depressão, a enxaqueca, a periodontite, o Lúpus Eritematoso Sistêmico e a Doença do Refluxo Gastresofágico. Todavia, tais fatores excêntricos estão associados a doenças pregressas, caracteristicamente, causais no contexto brasileiro, como diabetes mellitus, hipertensão arterial, obesidade e tabagismo, bem como a fatores relacionados ao clima local e hábitos alimentares inadequados. Este estudo possuiu como limitação a escassez de artigos de níveis de evidência 1 e 2, e produções nacionais. 


\section{REFERÊNCIAS}

1. Organização Pan-Americana da Saúde/Organização Mundial da Saúde. Doenças cardiovasculares. [acesso em: 16 de agosto de 2018]. Disponível em: https://www.paho.org/bra/index.php?option=com_content\&view=article\&id=5253:doencas-cardiovasculares\&ltemid $=839$.

2. Gus I, Ribeiro RA, Kato S, Medina C, Zazlavsky C, et al. Variações na prevalência dos fatores de risco para doença arterial coronariana no Rio Grande do Sul: uma análise comparativa entre 2002-2014. ArqBrasCardiol. 2015; 105(6): 573-579. Disponível em: http://dx.doi.org/10.5935/abc.20150127.

3. Brunori EHFR, Lopes CT, Cavalcante AMRZ, Santos VB, Lopes JL, Barros ALBL. Associação de fatores de risco cardiovasculares com as diferentes apresentações da síndrome coronariana aguda. Rev. Latino-Am. Enfermagem. 2014; 22(4): 538-46. Disponível em: http://dx.doi.org/10.1590/0104-1169.3389.2449.

4. Ribeiro KRA, Silva LP, Lima MLS. Conhecimento do infarto agudo do miocárdio: implicações para assistência de enfermagem. RevEnferm UFPI [internet]. 2016; 5(4): 63-8. Disponível em: http://www.ojs.ufpi.br/index.php/ reufpi/article/view/5546/pdf.

5. Smeltzer SC, Bare BG, Hinkle JL, Cheever KH. Brunner\&Suddarth, tratado de enfermagem médico-cirúrgica Volume 2. 12ª ed. Rio de Janeiro: Guanabara Koogan; 2014.

6. Medeiros TLF, Andrade PCNS, Davim RMB, Santos NMG. Mortalidade por infarto agudo do miocárdio. Revista de Enfermagem UFPE Online, Recife. 2018, fev; 12(2): 565-572. Disponível em: https://doi.org/10.5205/ 1981-8963-v12i2a230729p565-572-2018.

7. Soares CB, Hoga LAK, Peduzzi M, Sangaleti C, Yonekurra T, Silva DRAD. Revisão integrativa: conceitos e métodos utilizados na enfermagem. RevEscEnferm USP, São Paulo. 2014; 48(2): 335-345. Disponívelem: https://doi. org/10.1590/S0080-6234201400002000020.

8. The Ottawa Hospital Research Institute [Internet]. The Newcastle-Ottawa Scale (NOS) for assessing the quality if nonrandomized studies in meta-analyses. Disponível em: http://www.ohri.ca/programs/clinical_epidemiology/ 
oxford.htm. Acesso em: 17 jun. 2019

9. Moher D, Liberati A, Tetzlaff J, Altman DG, The PRISMA Group. Preferred Reporting Items for Systematic Reviews and Meta-Analyses: The PRISMA Statement. Epidemiol. Serv. Saúde, Brasília. 2015, abr-jun; 24(2). DOI: $10.5123 / S 1679-49742015000200017$.

10. Cortés JTA, Hernández VB, Hechavarría GAP, Duany OA, Carrión MEB. Factores de riesgo coronarios asociados al infarto agudo del miocardio en el adulto mayor. MEDISAN. 2013 [citado 11 de Feb 2013]; 17(1): 54-60. Disponível em: http://scielo.sld.cu/scielo.php?script=sci_arttext\&pid=S102930192013000100008\&lng=es.

11. Górski B, Nargiello E, Grabowska E, Opolski G, Gorska R. The Association Between Dental Status and Risk of Acute Myocardial Infarction Among Poles: Case-control Study. AdvClinExpMed, Polônia. 2016; 25(5), 861-870. Disponível em: http://dx.doi.org/10.17219/acem/58866.

12. Aviña-zubieta A, Fergus T, Vostretsova K, Vera M, Sayre E, Esdaile J. Risk of Myocardial Infarction and Stroke in Newly Diagnosed Systemic Lupus Erythematosus: A General Population-Based Study. Arthritis Care Res, Hoboken. 2017 Jun; 69(6): 849-856. Disponívelem: http://dx.doi.org/10.1002/acr.23018. 